\title{
Gravitational Search Algorithm and Selection Approach for Optimal Distribution Network Configuration Based on Daily Photovoltaic and Loading Variation
}

\author{
Koong Gia Ing, ${ }^{1}$ H. Mokhlis, ${ }^{1,2}$ H. A. Illias, ${ }^{1,2}$ M. M. Aman, ${ }^{1}$ and J. J. Jamian ${ }^{3}$ \\ ${ }^{1}$ Department of Electrical Engineering, Faculty of Engineering, University of Malaya, 50603 Kuala Lumpur, Malaysia \\ ${ }^{2}$ University of Malaya Power Energy Dedicated Advanced Centre (UMPEDAC), Level 4, Wisma R\&D UM, \\ Jalan Pantai Baharu, University of Malaya, 59990 Kuala Lumpur, Malaysia \\ ${ }^{3}$ Faculty of Electrical Engineering, Universiti Teknologi Malaysia, 81310 Johor Bahru, Johor, Malaysia \\ Correspondence should be addressed to H. Mokhlis; hazli@um.edu.my
}

Received 13 March 2015; Revised 14 June 2015; Accepted 21 June 2015

Academic Editor: Mengqi Hu

Copyright (C) 2015 Koong Gia Ing et al. This is an open access article distributed under the Creative Commons Attribution License, which permits unrestricted use, distribution, and reproduction in any medium, provided the original work is properly cited.

\begin{abstract}
Network reconfiguration is an effective approach to reduce the power losses in distribution system. Recent studies have shown that the reconfiguration problem considering load profiles can give a significant improvement on the distribution network performance. This work proposes a novel method to determine the optimal daily configuration based on variable photovoltaic (PV) generation output and the load profile data. A good combination and coordination between these two varying data may give the lowest power loss in the system. Gravitational Search Algorithm (GSA) is applied to determine the optimum tie switches positions for 33-Bus distribution system. GSA based proposed method is also compared with Evolutionary Programming (EP) to examine the effectiveness of GSA algorithm. Obtained results show that the proposed optimal daily configuration method is able to improve the distribution network performance in term of its power loss reduction, number of switching minimization and voltage profile improvement.
\end{abstract}

\section{Introduction}

Over the last few decades, the power loss of distribution system has raised attention of researchers to increase the efficiency of the existing network. Different researchers have proposed different approaches for minimization of power system losses [1]. One of the common approaches is through network reconfiguration.

Network reconfiguration is defined as altering the topological structures of the distribution feeders, by changing the position of tie and sectionalizing switches; however, under normal operation, medium voltage distribution networks operate in radial manner $[2,3]$. During normal condition, network reconfiguration is used to improve the power quality, maintain the voltage stability, and reduce power loss, while for fault condition it is used for service restoration. Once the source of fault is removed by opening all the switches surrounding the affected portions, the service can be restored within a very short time.
In the past, various algorithms have been proposed for network reconfiguration. Merlin and Back were the pioneers in proposing network reconfiguration as a tool for power loss reduction [4] in 1975. The network reconfiguration problem was solved by closing all tie switches in the system (resulting in mesh network); then the switches are successively opened, one at a time until a new radial network with minimum power losses is obtained. Later on, Shirmohammadi and Hong improved the Merlin method by avoiding the approximation made in [4]. Shirmohammadi theorem starts by closing all tie switches which are then opened by anther based on optimum power flow results [5]. This method was also found efficient in terms of computation time as compared to Merlin method. Reference [2] introduced the "feeder exchange approach" and calculated the loss reduction due to load transfer between two feeders. A year later, Baran and Wu improved the branch exchange method [2] by proposing two approximated power flow equations and solved the problem of loss minimization and loss reduction as an integer 
programming problem [6]. In the same year, Lin and Chin [7] have considered service restoration, in addition to loss minimization in solving network reconfiguration problem. Zhu et al. performed the network reconfiguration based on modified heuristic solution and experience system operation rules [8]. In a latest research, Mohd Zin et al. in [9] have used the heuristic search algorithm to find the minimum power losses as an objective function, introduced by the author in [6]. The update principle of heuristic search is made by finding the branch having minimal current, till the optimum solution is obtained.

Most of the methods discussed above require exhaustive search mechanism by considering all possible solutions from a predetermined set and subsequently pick the best one. However, such methods are considered as nonefficient in terms of computation time and space requirement. Intelligent, greedy, and nature observed heuristic techniques have also been proposed in the literature and they are commonly known as Artificial Intelligent (AI) methods [10]. Artificial Intelligent methods are a special class of heuristic search methods [10]. Intelligent based optimization methods have also been utilized in finding optimum tie switch combinations in network reconfiguration problem. Authors have used Genetic Algorithms (GA) [11], fuzzy [12-14], neural network $[15,16]$, fuzzy-GA [17], Particle Swarm Optimization (PSO) [18], Matroid Theory [19], Hybrid Evolutionary algorithm [20], Ant Colony Search (ACS) algorithm [21, 22], Harmony Search Algorithm [23], and Bacterial Foraging Algorithm [24]. Das [12] and Savier and Das [25] have proposed fuzzy based multiobjective approach for loss reduction and considered voltage limit and line limits as well in fuzzy set. Penalty based multiobjective-single-fitness approaches have been utilized in [21], to combine power losses with system constraints including voltage limits and line limit. The multiobjective problem is solved using ACS algorithm.

Besides network reconfiguration, Distributed Generation (DG) such as minihydro, photovoltaic, and wind is installed in distribution system to solve the power loss problem due to voltage drop. However, the placement and size of DG are essential as they have a great impact on the power loss [26]. Many researchers have proposed different methods for optimum DG location and size separately [26-28] or simultaneously $[29,30]$. However, all the aforementioned works have considered a constant load and fixed DG output. In practical, load pattern and DG generation output dynamically change with time. Therefore, some studies have also considered a variable load pattern in their researches. For example, a gradual approaching method is proposed in [31] to solve dynamic reconfiguration. In [32], researchers solved the network reconfiguration problem with daily variable load implementation as well. These studies have proven that the network performance was improved when the time-varying load profiles were taken into consideration.

Apart from that, there are few works on network reconfiguration with the presence of DG considering variable load profile. For example, network reconfiguration with the presence of wind and photovoltaic generation for total daily power loss reduction using improved genetic algorithm is proposed in [33]. Network reconfiguration with photovoltaic generation for power loss reduction considering the DG penetration and three different load levels is presented in [34].

In this work, a novel method is proposed to solve the network reconfiguration problem based on daily PV generation output and variable load profiles. Gravitational Search Algorithm (GSA) proposed in [35] is used in this paper to determine the best daily system configuration. The proposed method can be viewed in two parts. In the first part, hourly optimal configuration considering the PV generation and load profile is carried out using GSA. In the second part, a selective approach is used to determine the best configuration that gives the lowest power loss for the whole day $(24 \mathrm{hrs})$ or optimal daily configuration. The proposed method is implemented on 33-bus distribution system. The proposed method results are also compared with Evolutionary Programming (EP) to show the effectiveness of GSA.

This paper is organized as follows. In Section 2, mathematical model for network reconfiguration is described. The proposed method including application of GSA and selection approach is provided in Section 3. Selection of PV module is presented in Section 4 while load modelling and PV generation are presented in Section 5. In Section 6, analysis of the performance of the proposed method is discussed. Finally, the conclusions are presented in Section 7.

\section{Mathematical Model for Distribution Network Reconfiguration Problem}

Network reconfiguration is used to minimize the distribution system power loss. The new configuration selected must fulfil the constraints and maintain the radial structure of the distribution system. In this study, the main objective is to gain minimum power loss of the distribution system. Thus, the objective function can be formulated as follows:

$$
\operatorname{Min}\left\{P_{\text {loss }}=\sum_{h}^{T} \sum_{f=1}^{N}\left|I_{f}\right|^{2} l_{f} R_{f}\right\} \text {, }
$$

where $N$ is total line in the system. $I_{f}$ is real active current of line $f . R_{f}$ is resistance of line $f . f$ is line number. $l_{f}$ is the topology status of line $f(1=$ close, $0=$ open $)$.

$T$ is the total hour considered in the time frame and $h$ is the current time considered. In this work, (1) is used to analyse the power loss for every hour $(h)$ of a day with the load data of hour $(h)$ and PV generation of hour $(h)$.

The power system constraints that are also being considered in the study are as follows.

(a) Voltage Bus Constraint. The value of the bus voltage $V_{\text {bus }}$ at each node must be within the acceptable limits to maintain the power quality. For example, in this work, the minimum voltage $\left(V_{\min }\right)$ used is 0.95 p.u. and maximum voltage $\left(V_{\max }\right)$ is 1.05 p.u.:

$$
V_{\text {min }}<V_{\text {bus }}<V_{\text {max }}
$$


(b) Radial Configuration Constraint. The radial configuration of the network must be maintained after the reconfiguration process. In order to ensure this requirement, graph theory is used to determine the radiality of the network. In this work, a MATLAB function is used to check the radiality of a network as follows:

$$
\mathrm{TF}=\text { graphisspantree }(G),
$$

where $G$ is the distribution system. If the network is radial, TF equals 1 (true); else it is 0 (false). A radial distribution system must connect all the buses and it contains no loop. In order to maintain the radial structure of the distribution system, a network with loop form, one of the switches from that loop must be opened to retain the radial structure.

There are several methods for radial power flow analysis such as backward-forward method. However, in this work the proposed method uses Newton-Raphson load flow for power flow analysis to calculate the power loss. This load flow is selected to avoid fussy requirements on radial structure and prevent complicated computation [36]. It can handle the DG in the calculation more effectively as compared to other distribution load flow methods. Furthermore, this method provides high convergence with low storage required which can be easily applied with any metaheuristic optimization method.

The real power of bus $f, P_{f}$, reactive power of bus $f, Q_{f}$, the differences in real power, $\Delta P_{f}$, the differences in reactive power, $\Delta Q_{f}$, rectangular Newton-Raphson, and power loss can be computed as

$$
\begin{aligned}
P_{f}= & \sum_{k=1}^{N}\left|V_{f}\right|\left|V_{k}\right|\left|Y_{f k}\right| \cos \left(\theta_{f k}-\delta_{f}+\delta_{k}\right), \\
Q_{f}= & \sum_{k=1}^{N}\left|V_{f}\right|\left|V_{k}\right|\left|Y_{f k}\right| \sin \left(\theta_{f k}-\delta_{f}+\delta_{k}\right), \\
\Delta P_{f}= & P_{f}^{\mathrm{sp}}-P_{f}, \\
\Delta Q_{f}= & Q_{f}^{\mathrm{sp}}-Q_{f}, \\
{\left[\begin{array}{c}
\Delta P \\
\Delta Q
\end{array}\right]=} & {\left[\begin{array}{ll}
\frac{\partial P}{\partial \delta} & \frac{\partial P}{\partial V} \\
\frac{\partial Q}{\partial \delta} & \frac{\partial Q}{\partial V}
\end{array}\right]\left[\begin{array}{c}
\Delta \partial \\
\Delta|V|
\end{array}\right], } \\
P_{\mathrm{loss}}= & \sum_{f=1}^{N} A_{f k}\left(P_{f} P_{k}+Q_{f} Q_{k}\right) \\
& +B_{f k}\left(Q_{f} P_{k}-P_{f} Q_{k}\right), \\
A_{f k}= & \frac{R_{f k} \cos \left(\delta_{f}-\delta_{k}\right)}{V_{f} V_{k}}, \\
B_{f k}= & \frac{R_{f k} \sin \left(\delta_{f}-\delta_{k}\right)}{V_{f} V_{k}},
\end{aligned}
$$

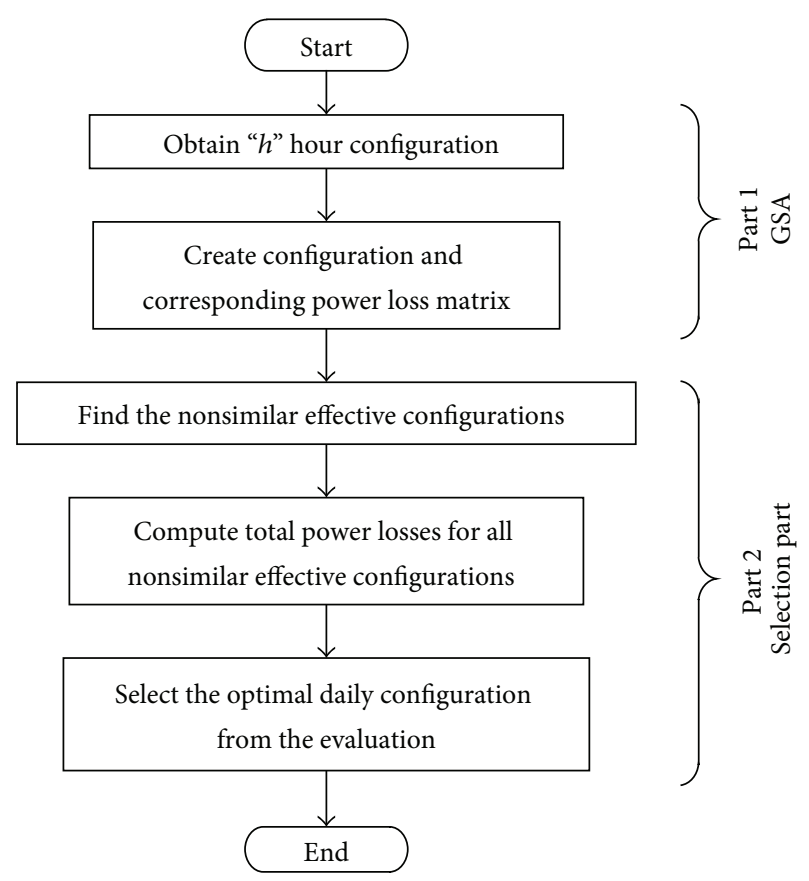

FIGURE 1: Flowchart of the proposed method.

where $V_{f}$ and $V_{k}$ are voltage magnitude of bus $f$ and bus $k$, respectively, $Y_{f k}$ and $\theta_{f k}$ are magnitude and angel of $Y_{f k}$ element in the bus admittance matrix, respectively, $\delta_{f}$ and $\delta_{k}$ are voltage angel of bus $f$ and bus $k$, respectively, $P_{f}^{\mathrm{sp}}$ and $Q_{f}^{\mathrm{sp}}$ are specified real and reactive power at bus $f$, respectively, $P_{k}$ and $Q_{k}$ are real and reactive power of bus $k$, respectively, and $R_{f k}$ is line resistance between bus $f$ and bus $k$.

\section{Proposed Method}

The proposed method for finding the best configuration based on daily PV generation and load profiles is presented in this section. The proposed algorithm consists of two main parts, as shown in Figure 1. In the first part, a day is divided into $h$ hours, where $h$ equals 24 hours in this work. Using GSA and graph theory, optimal configuration is obtained for each hour while the distribution network is maintained to be radial. Hence, a total of $h$ effective configurations are obtained for the network. In part two, the optimal daily configuration is obtained with a selective approach.

Part 1: Finding Effective Configuration Using Gravitational Search Algorithm (GSA). Gravitational Search Algorithm (GSA) is first introduced by Rashedi et al. in [35]. GSA is the newest stochastic search algorithm which is based on Newtonian laws and mass interactions. In this algorithm, the population individuals are referred to as masses and their performances are measured by their position masses. Each mass will have four particulars: its position, its inertial mass, its active gravitational mass, and passive gravitational mass. The position of the mass represented a solution while its gravitational and inertial masses are corresponding to the fitness function. According to Newtonian laws, all these objects will attract each other due to the gravity force. Due to 
this force, all these objects will move towards the object with heavier mass. In other words, heavy masses equaled good solutions and they move slower than the lighter masses that equaled bad solutions. In this way, the exploitation step of the algorithm is guaranteed.

The details of the proposed method to reduce power loss are based on the following steps.

Step 1 (input data and parameters). Input data are inserted in the program. This includes bus data, line data, PV output, base MVA, base voltage, predefined voltage bus range, radial configuration constraint, maximum iteration, and accuracy. The parameter needed to be set in GSA is the number of masses $\left(N_{\text {mass }}\right)$.

Step 2 (generating initial masses). The initial population is determined by selecting random switches to be opened in the distribution network to form the masses. The number of switches to be opened is $N_{\text {opened }}$. The set of switches to be opened is written as follows:

$$
\text { Mass }_{i}=\left\lfloor S_{1}^{1}, S_{2}^{2}, \ldots, S_{N_{\text {opened }}}^{d}\right\rfloor \text { for } i=1,2, \ldots, N_{\text {mass }},
$$

where Mass $_{i}$ represents the position of $i$ th mass in the $d$ th dimension or $d$ th component in ith mass. $S_{1}^{1}, S_{2}^{2}$, and $S_{N_{\text {opened }}}^{d}$ are the switches to be opened in $d$ th dimension. Only the set of switches to be opened that satisfy all the constraints will be generated as the mass. At the end of this step, all the elements in the masses for network reconfiguration are then converted to whole number by rounding the decimal number to the nearest positive number.

Step 3 (evaluate fitness of each mass). In the fitness function, power loss is calculated in the current mass population using Newton-Raphson load flow analysis.

Step 4 (update gravitational constant, best mass, worst mass, and inertia mass of each iteration). In order to control the searching accuracy, the gravitational constant, $G$, is initialized at the beginning and is reduced with iteration. Hence, gravitational constant $G$ is a function of initial value of gravitational constant, $G_{o}$, and iteration, iter, as follows:

$$
G(\text { iter })=G_{o} \ell^{-\alpha(\text { iter/max _iter })},
$$

where $\alpha$ is a user specified constant, iter is the current iteration, and max_iter is the total number of iterations.

The active gravitational mass, $M_{a}$, passive gravitational mass, $M_{p}$, and inertial mass of mass $i, M_{i i}$, are computed using fitness evaluation. According to Newton's law and law of motion, a heavier mass has higher attractions and move more slowly. Hence, in GSA, a heavier mass is represented as good solution and the pattern of movement is represented by the explorations. The inertia mass, $M_{i}$, is updated as follows by assuming all masses are equal:

$$
\begin{aligned}
M_{a i} & =M_{p i}=M_{i i}=M_{i}, \\
m_{i}(\text { iter }) & =\frac{\text { fit }_{i}(\text { iter })-\text { worst }(\text { iter })}{\text { best }(\text { iter })-\text { worst }(\text { iter })},
\end{aligned}
$$

$$
M_{i}(\text { iter })=\frac{m_{i} \text { (iter) }}{\sum_{j=1}^{N_{\text {mass }}} m_{j}(\text { iter })},
$$

where fit $_{i}$ (iter) represents the power loss of the mass $i$ at iteration, iter, and best(iter) and worst(iter) represent the strongest and weakest masses with respect to the lowest and highest power losses in current iteration. The mass $j$ of current iteration is represented as $m_{j}$ (iter). For minimization problem, the best(iter) and worst(iter) are defined as

$$
\begin{aligned}
\text { best (iter) } & =\min _{j \in\left\{1, \ldots, N_{\text {ma }}\right\}} \text { fit }_{j} \text { (iter), } \\
\text { worst (iter) } & =\max _{j \in\left\{1, \ldots, N_{\text {mass }}\right\}} \text { fit }_{j} \text { (iter). }
\end{aligned}
$$

Step 5 (calculation of the total force in different directions). The gravitational force, $F_{i j}$, of mass $i$ due to mass $j$ at current iteration, iter, can be computed as follows:

$$
\begin{aligned}
& F_{i j}(\text { iter })=G(\text { iter }) \frac{M_{i}(\text { iter }) \times M_{j}(\text { iter })}{R_{i j}(\text { iter })+\varepsilon}\left(\text { Mass }_{j}(\text { iter })\right. \\
& \left.\quad-\operatorname{Mass}_{i}(\text { iter })\right)
\end{aligned}
$$

where $M_{i}$ is the inertial mass of the mass $i, M_{j}$ is the inertial mass of mass $j, \varepsilon$ is a small constant, and $R_{i j}$ (iter) is the Euclidian distance between $i$ and $j$ masses specified as follows:

$$
R_{i j}(\text { iter })=\| \operatorname{Mass}_{i}(\text { iter }), \text { Mass }_{j}(\text { iter }) \|_{2} .
$$

Step 6 (calculation of acceleration and velocity). The acceleration of the mass $i$ at current iteration, iter, in $d$ th dimension, and $a_{i}^{d}$ (iter) is defined as follows:

$$
a_{i}^{d}(\text { iter })=\frac{F_{i}^{d}(\text { iter })}{M_{i i}(\text { iter })}
$$

where $F_{i}^{d}$ (iter) is the total force that acts on mass $i$ of $d$ th dimension and is calculated as follows:

$$
F_{i}^{d}(\text { iter })=\sum_{j \in k_{\text {best }}, j \neq i}^{N_{\text {mass }}} \operatorname{rand}_{j} F_{i j}^{d} \text { (iter). }
$$

The random number between interval $[0,1], \operatorname{rand}_{j}$ is introduced in GSA. In order to compromise between exploration and exploitation in this algorithm, the exploration must fade out and exploitation must fade in with lapse of iterations. In other words, all masses apply force to each other in the beginning and only one mass applies force to others in the end of the algorithm. Based on the aforementioned concept, $K_{\text {best }}$, a function of iteration which is the set of first $K$ masses with the lowest power loss and biggest mass, is introduced to this algorithm. $K_{\mathrm{o}}$, the initial value of $K_{\text {best }}$, is set at the beginning and decreased with iterations. Thus, $K_{\text {best }}$ is decreased linearly with iterations as well. In this way, a smaller value of $K_{\text {best }}$ will result in less interaction between the masses by gravitational force. Hence, the movement and computational of power loss 
are reduced and lead to convergence [37]. The next velocity of a mass is given as follows:

$$
v_{i}^{d}(\text { iter }+1)=\operatorname{rand}_{i} \times v_{i}^{d}(\text { iter })+a_{i}^{d}(\text { iter }) .
$$

Step 7 (update masses' position). The next position of a mass can be expressed as follows:

$$
\operatorname{Mass}_{i}^{d}(\text { iter }+1)=\operatorname{Mass}_{i}^{d}(\text { iter })+v_{i}^{d}(\text { iter }+1) .
$$

Step 8 (convergence). Steps 3 to 8 are repeated until the stopping criterion is reached.

Step 9 (matrix of $h$ feasible configurations). A matrix consists of $h$ feasible configuration and its power loss is created. Each configuration consists of $N_{\text {opened }}$ switch to be opened which depends on the chosen test system. Configuration $h$ is the best configuration with the minimum power loss at hour $h$. For example, configuration 1 is the best configuration at 0100 and so on as illustrated in

$$
\begin{array}{ccccccc} 
& S_{1} & S_{2} & S_{3} & \cdots & S_{N_{\text {opened }}} & \text { Power loss for } h \text { hour } \\
\text { Configuration 1 } \\
\text { Configuration 2 } \\
\text { Configuration } h \\
\vdots
\end{array}\left[\begin{array}{cccccc}
S_{1,1} & S_{1,2} & S_{1,3} & \cdots & S_{1, N_{\text {opened }}} & P_{\text {loss }} s_{1} \\
S_{2,1} & S_{2,2} & S_{2,3} & \cdots & S_{2, N_{\text {opened }}} & P_{\text {loss }} s_{2} \\
\vdots & \vdots & \vdots & \vdots & \vdots & \vdots \\
S_{h, 1} & S_{h, 2} & S_{h, 3} & \cdots & S_{h, N_{\text {opened }}} & P_{\text {loss }} s_{h}
\end{array}\right] .
$$

Part 2: A Selective Approach for Optimal Daily Configuration. In the steps above, $N_{z}$ number of configurations will be obtained for total time, $T$. Since most of the PV generations and the load of certain $h$ hour in total time, $T$, are approximately the same, most of the obtained configurations in (22) are similar. The similar configurations, $N_{\text {similar }}$, are removed from the matrix. Hence, only the nonsimilar effective configurations are left in the matrix, $N_{\text {left }}\left(N_{z}-N_{\text {similar }}=N_{\text {left }}\right)$.
The first nonsimilar effective configuration in the matrix represents the set switch 1, and so forth, until the last nonsimilar effective configuration, and set switch $y$. Hence, a

\begin{tabular}{|c|c|}
\hline Set Switch 1 & {$\left[X_{1}\right.$} \\
\hline Set Switch 2 & $X_{2}$ \\
\hline$\vdots$ & \\
\hline Set Switch $y$ & $X_{y}$ \\
\hline
\end{tabular}
matrix consists of all nonsimilar effective configurations and is obtained as illustrated in

Power loss for each set switch at $h$ hour

Each set switch obtained in Part 2 is used to calculate the total power loss for a day using Newton-Raphson load flow analysis. Then, the total daily power losses of the distribution system for all sets of the switch are obtained and the switch set with the minimum total daily power loss is selected as the optimum configuration of the day, as illustrated in

Set switch Total daily power loss

$$
\left[\begin{array}{cc}
X_{1} & \text { TotDaily } P_{\text {loss }} X_{1} \\
X_{2} & \text { TotDaily } P_{\text {loss }} X_{2} \\
\vdots & \vdots \\
X_{y} & \text { TotDaily } P_{\text {loss }} X_{y}
\end{array}\right] \text {, }
$$

where optimal daily configuration $=\min \left(\right.$ TotDaily $\left.P_{\text {loss }}\right)$.

\section{Selection of PV Module}

The PV generation in this study is dependent on the solar irradiance and ambient temperature of Malaysian Meteorological Department for year 2008 at Kuantan site. The capacity factor (CF) is calculated using four PV modules from [38] based on the solar irradiance mentioned as above for a day. As shown in Figure 2, module D has the highest CF (0.2223); therefore, module D is selected for this study. The PV output for 10000 units and each $h$ time interval are calculated using (8) to (12). The PV generation is shown in Figure 3:

$$
\begin{aligned}
& T_{\mathrm{CZ}}(h)=T_{a}+s_{\mathrm{az}}(h r)\left(\frac{N_{\mathrm{OT}}(\text { module })-20}{0.8}\right), \\
& I_{Z}(h)=s_{\mathrm{aZ}}(h) \\
& \quad \cdot\left[I_{\mathrm{SC}}(\text { module })+K_{i}(\text { module })\left(T_{\mathrm{CZ}}(h)-25\right)\right],
\end{aligned}
$$




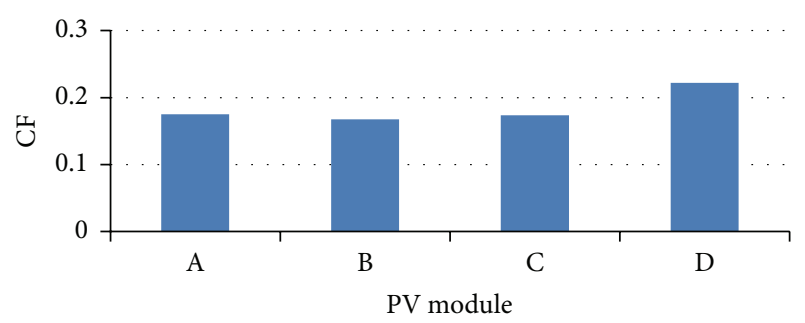

FIGURE 2: CF of the PV module.

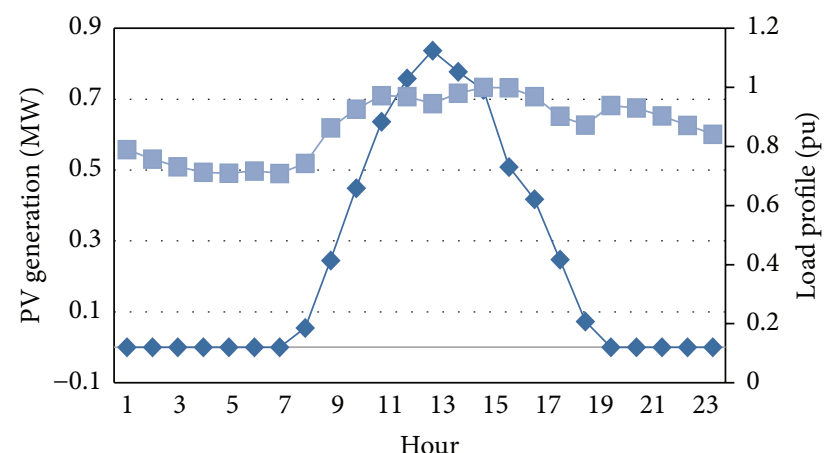

$\checkmark$ PV generation

- Load profile

FIGURE 3: PV generation and load profile of a day.

$$
\begin{aligned}
& V_{Z}(h)=V_{\mathrm{OC}}(\text { module })-\left(K_{V}(\text { module }) \times T_{\mathrm{CZ}}(h)\right), \\
& \mathrm{FF}(\text { module })=\frac{\left(V_{\mathrm{MPP}}(\text { module }) \times I_{\mathrm{MPP}}(\text { module })\right)}{V_{\mathrm{OC}}(\text { module }) \times I_{\mathrm{SC}}(\text { module })}, \\
& P_{\mathrm{SZ}}(h)=N_{\mathrm{PV}} \times \mathrm{FF}(\text { module }) \times V_{Z}(h) \times I_{Z}(h),
\end{aligned}
$$

where $T_{\mathrm{CZ}}(h)$ is cell temperature $\left[{ }^{\circ} \mathrm{C}\right]$ during $h$ current time, $T_{a}$ is ambient temperature $\left[{ }^{\circ} \mathrm{C}\right], K_{V}$ (module) is voltage temperature coefficient of the $\mathrm{PV}$ module $\left[\mathrm{V} /{ }^{\circ} \mathrm{C}\right], K_{i}$ (module) is current temperature of the PV module $\left[\mathrm{A} /{ }^{\circ} \mathrm{C}\right], N_{\mathrm{OT}}$ (module) is nominal operating temperature of cell of the PV module $\left[{ }^{\circ} \mathrm{C}\right], \mathrm{FF}$ (module) is fill factor of the PV module, $I_{\mathrm{SC}}$ (module) is short circuit current of the PV module $[\mathrm{A}], V_{\mathrm{OC}}$ (module) is open circuit voltage of the PV module [V], $I_{\mathrm{MPP}}$ (module) is current at maximum power point of the PV module [A], $V_{\mathrm{MPP}}$ (module) is voltage at maximum power point of the PV module [V], $P_{\mathrm{SZ}}(h)$ is output generation of PV module during $h$ current time, and $s_{\mathrm{az}}(h)$ is average solar irradiance during $h$ current time.

\section{Load Modelling and PV Generation}

The load profiles were received from Tenaga Nasional Berhad (TNB) [39] as shown in Figure 3. This study provides hourly peak load per unit of the daily peak load.

\section{Analysis of Proposed Method}

The test system for this study consists of $12.66 \mathrm{kV} 33$-bus radial distribution. The single line diagram of the system is shown in Figure 2. The system consists of three DGs, five labelled tie switches, 32 unlabelled sectionalizing switches, and 33 labelled buses. The line between bus 1 and bus 2 is referred to as switch 1 , line between bus 2 and bus 3 is referred to as switch 2 , and so forth until switch 32 . The five tie switches are referred to as switches $33,34,35,36$, and 37 as shown in Figure 4. The line and load data are taken from [6]. For analysis purpose, the DGs are assumed to be installed at buses 6,18 , and 22 . However, the locations can be changed based on the actual site data or using other placement methods such as in [29]. The parameters of GSA used in the simulation are set to $N_{\text {mass }}=100$, max_iter $=100$, and $G_{0}=$ sum of all active power in the system and $\alpha=10$. The simulations are carried out on a computer with Intel Duo Core, 3.0 GHz, and 3.0 GB RAM.

The $S_{\text {base }}$ equals $100 \mathrm{MVA}$ and all the calculations are performed per unit. In the simulation of the network, four scenarios were considered to analyse the capability of the proposed method. They are as follows.

Scenario 1. The system is without reconfiguration and variation of the load and PV generation are considered.

Scenario 2. The system is with optimal hourly reconfiguration and variation of the load and PV generation are considered.

Scenario 3. The system is with optimal daily configuration obtained from the proposed method.

Scenario 4. The system is with optimal daily configuration obtained by considering 24 demand levels and PV generation as whole.

The results of a matrix consist of $h$ feasible configurations and their power loss obtained in Part 1 of the study is shown in Table 4 . The highlighted configurations in Table 4 represent repeated configurations that will be removed in selection approach in Part 2 to obtain the nonsimilar effective configurations matrix in Part 2. The remaining are the nonimilar effective configurations. The results of nonsimilar effective configurations matrix in Part 2 of the study are shown in Table 5.

Then, for each set of the switch in Table 5, their total daily power loss is obtained by carrying out Newton-Raphson load flow analysis. The results of the total power loss evaluation for all sets switch obtained in Part 2 are shown in Table 1 .

From Table 1, the total daily power loss of set switch 19 which is represented with $*$ is the lowest among the 20 sets switch. Even set switch 16 with the lowest power loss at 1300 does not yield the lowest total daily power losses. Hence, set switch 19 is chosen as the optimal daily configuration which is the best configuration for the day. The real results of the four scenarios are summarised in Table 2. The proposed method is carried out using EP for these four scenarios as well as for comparison as shown in 


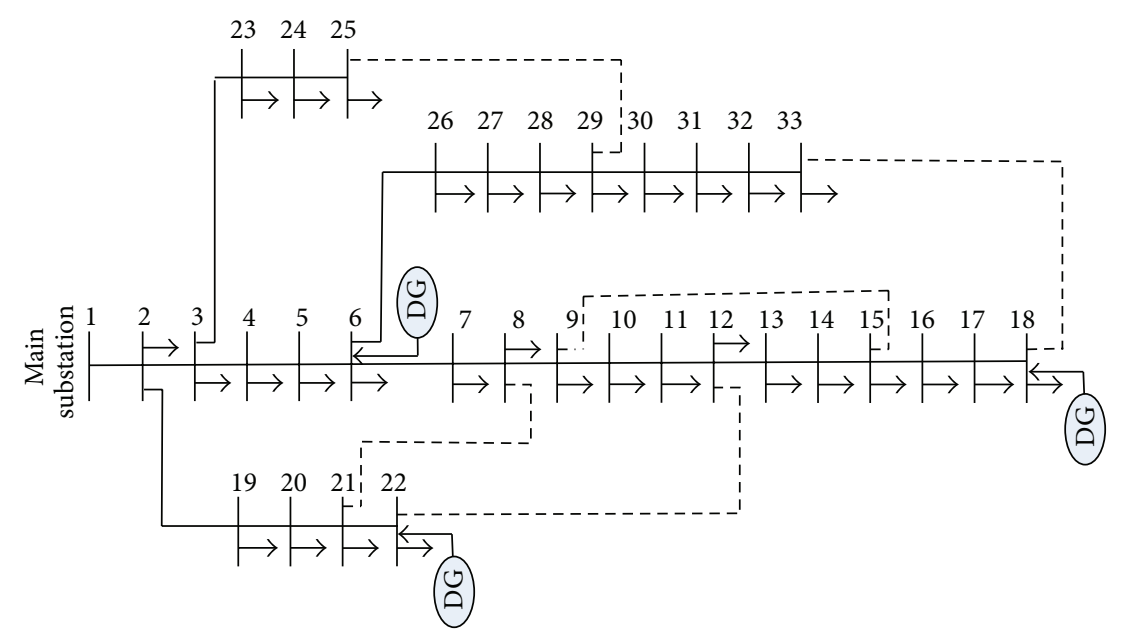

FIGURE 4: 33-bus test system with DG at buses 6, 18, and 22.

TABLE 1: Results of total power loss evaluation for all sets switch obtained in Part 2.

\begin{tabular}{|c|c|c|c|c|c|c|c|c|c|c|}
\hline Set switch & 1 & 2 & 3 & 4 & 5 & 6 & 7 & 8 & 9 & 10 \\
\hline $\begin{array}{l}\text { Total daily } \\
\text { power loss }(\mathrm{kW})\end{array}$ & 2724.30 & 2770.07 & 2638.54 & 2752.83 & 2960.39 & 2564.08 & 2219.49 & 2909.31 & 2375.65 & 2149.08 \\
\hline Set switch & 11 & 12 & 13 & 14 & 15 & 16 & 17 & 18 & $19^{*}$ & 20 \\
\hline $\begin{array}{l}\text { Total daily } \\
\text { power loss }(\mathrm{kW})\end{array}$ & 2249.01 & 2303.23 & 2330.55 & 3202.01 & 2088.32 & 2256.25 & 2721.87 & 2256.25 & 2075.51 & 2473.59 \\
\hline
\end{tabular}

Table 2. EP is selected for comparison since it is a common and well-established optimization method. The optimal daily configuration obtained in Scenario 4 is 37, 10, 7, 13, and 17 with GSA and 6, 8, 17, 10, and 5 with EP.

From Table 2, the total daily power loss is reduced by $50.36 \%$ in Scenario 2, $48.40 \%$ in Scenario 3, and $48.02 \%$ in Scenario 4 using GSA as optimizing tool and $44.77 \%$ in Scenario 2, 41.97\% in Scenario 3, and $41.00 \%$ in Scenario 4 using EP as optimizing tool compared with Scenario 1 with same load profile and PV generation. The total daily power loss reduction using Scenario 2 is $1.96 \%$ (GSA) and $2.80 \%$ (EP) more compared with Scenario 3 and $2.34 \%$ (GSA) and 3.77\% (EP) more compared with Scenario 4. However, no switching is required for Scenarios 3 and 4. For example, 24 times switching are requested in Scenario 2 while no switching is requested in Scenarios 3 and 4. Scenarios 3 and 4 far outweigh Scenario 2 with an acceptable total power loss reduction less than Scenario 2. Apart from that, Scenario 3 gives slightly higher total daily power loss reduction compared with Scenario 4 . This is because more constraints need to be considered when 24 demand levels and PV generation are considered as whole. Hence, this limited the solution options.

The DG installation location and number are assumed in this work. Altering DG installation location and number will automatically effect the power loss. The performance of the proposed method is not affected since the algorithm still the same just altering the load data. In other words, more power loss can be reduced with optimal DG installation location and number for a distribution network. An optimal daily configuration still can be obtained with the proposed method. Similarly for different type of DG with their typical output generation.

The performance of Scenario 2 is reduced when changing the hour slots from 1 hour to 2 hours or 4 hours and so forth. In this case, lower total daily power loss is obtained in Scenario 3 compared to Scenario 2 if the hour slot is increased. The total daily power loss for Scenarios 2 and 3 and optimal daily configuration obtained from Scenario 3 for hour slots of one, two, and four are presented in Table 3. It can be noticed that the total daily power loss in Scenario 3 is less than Scenario 2 for two- and four-hour slots. However, these results are the optimal results since the load demand and PV generation are changing with time. Therefore, the analysis in this work is carried out based on hourly basis.

From this analysis, it clearly shows that the proposed method is effective to determine optimal daily configuration based on PV generation and load profiles for a day. Apart from that, GSA with selection approach gives the best solution compared with EP with selection approach. Figure 5 illustrates the optimal configuration obtained from the proposed method.

The voltage profiles for all scenarios are shown in Figure 6. All the scenarios show the similar shape of the voltage profiles with slight change in the magnitude. The proposed method also improves the overall voltage profiles of the distribution system besides generating lower power loss.

From Figure 6, all bus voltages in Scenario 1 are below the 0.95 p.u. voltage constraint in a day. Although all the bus voltages satisfy the minimum voltage constraint only from 
TABLE 2: Comparison of the total daily power loss among the four scenarios.

\begin{tabular}{|c|c|c|c|c|}
\hline & $\begin{array}{l}\text { Scenario } 1 \text { (without } \\
\text { reconfiguration) }\end{array}$ & $\begin{array}{l}\text { Scenario } 2 \text { (with optimal } \\
\text { hourly reconfiguration) }\end{array}$ & $\begin{array}{l}\text { Scenario } 3 \text { (with } \\
\text { proposed method) }\end{array}$ & $\begin{array}{l}\text { Scenario } 4 \text { (with optimal } \\
\text { daily configuration of } 24 \\
\text { demand levels as whole) }\end{array}$ \\
\hline \multicolumn{5}{|c|}{ GSA and selection approach } \\
\hline $\begin{array}{l}\text { Total daily power loss } \\
(\mathrm{kW})\end{array}$ & 4022.30 & 1996.58 & 2075.51 & 2090.78 \\
\hline \multicolumn{5}{|c|}{ EP and selection approach } \\
\hline $\begin{array}{l}\text { Total daily power loss } \\
(\mathrm{kW})\end{array}$ & 4022.30 & 2221.52 & 2334.28 & 2373.17 \\
\hline
\end{tabular}

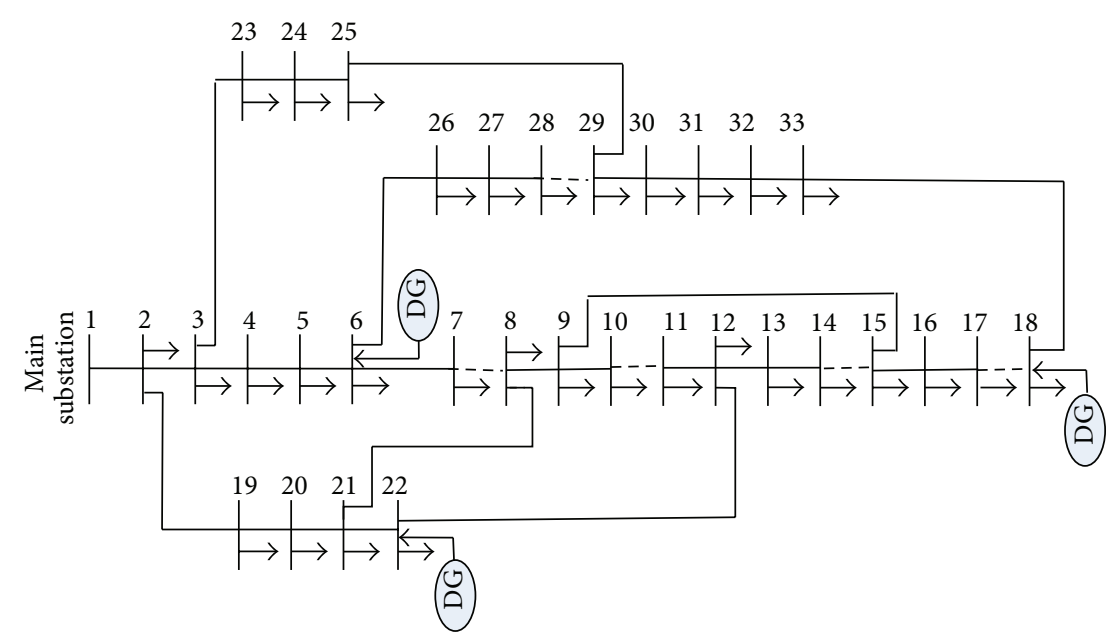

FIGURE 5: Optimal daily configuration.

TABLE 3: Comparison of the total daily power loss among one, two, and four hours' slots.

\begin{tabular}{lccc}
\hline $\begin{array}{l}\text { Hour slots } \\
(\mathrm{hr})\end{array}$ & Scenario 2 & Scenario 3 & $\begin{array}{c}\text { Optimal daily } \\
\text { configuration in } \\
\text { Scenario 3 }\end{array}$ \\
\hline 1 & 1996.58 & 2075.51 & $28,14,7,17,10$ \\
2 & 2204.75 & 2088.97 & $31,6,37,34,10$ \\
4 & 2132.67 & 2099.09 & $28,10,7,17,13$ \\
\hline
\end{tabular}

time 0200 to 1800 , the overall voltage profiles are improved clearly for all the buses in Scenarios 2, 3, and 4. The voltage profiles at time 1800 for all the buses for Scenarios 1, 2, 3, and 4 are shown in Figure 7.

From Figure 7, it can be seen that, at time 1800, the voltage profiles for Scenarios 1, 2, 3, and 4 are almost the same for all buses. Scenarios 2, 3, and 4 improve the voltage profiles of all the buses and the voltages are within acceptable range especially for buses 28 to 33 . This implies that reconfiguration considering PV generation and load profiles yields desired results of minimizing the power loss, reducing the number of switching and improving the voltage profiles. Meanwhile, Figure 8 shows the ability of the GSA with proposed method in finding the optimum configuration at times 0100, 0800, and 1800 which required less than 10 numbers of iterations to reach the optimal point. It is to note that the large the

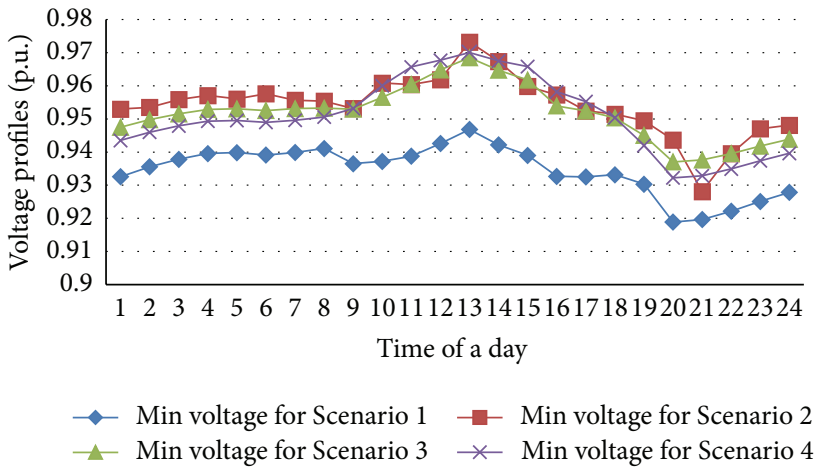

FIGURE 6: Performance of voltage profile for all scenarios of a day.

number of iterations, the longer computing time required as the iteration is counted based on the fixed looping in programming. Thus, in this work, the number of iterations is set to 100 .

\section{Conclusion}

In this work, a novel method to determine the best configuration based on the daily PV generation and load profiles in the distribution network has been successfully proposed. Experimental results indicate that the proposed method is 
TABLE 4: Results of matrix consists of $h$ feasible configurations and their power loss obtained in Part 1.

\begin{tabular}{|c|c|c|c|c|c|c|}
\hline $\begin{array}{l}\text { Current } \\
\text { time }(\mathrm{h})\end{array}$ & $S_{1}$ & $S_{2}$ & $S_{3}$ & $S_{4}$ & $S_{5}$ & $\begin{array}{c}\text { Power losses } \\
\text { for } h \text { hour } \\
(\mathrm{kW})\end{array}$ \\
\hline 01 & 11 & 7 & 27 & 32 & 14 & 88.56 \\
\hline 02 & 11 & 7 & 28 & 14 & 36 & 80.08 \\
\hline 03 & 28 & 14 & 33 & 9 & 32 & 75.39 \\
\hline 04 & 28 & 9 & 33 & 32 & 14 & 71.31 \\
\hline 05 & 9 & 34 & 37 & 32 & 7 & 70.74 \\
\hline 06 & 32 & 28 & 34 & 7 & 11 & 71.65 \\
\hline 07 & 33 & 36 & 10 & 28 & 34 & 71.21 \\
\hline 08 & 37 & 34 & 9 & 32 & 7 & 70.73 \\
\hline 09 & 37 & 17 & 9 & 14 & 7 & 75.63 \\
\hline 10 & 21 & 31 & 37 & 7 & 13 & 70.41 \\
\hline 11 & 11 & 14 & 30 & 7 & 28 & 70.01 \\
\hline 12 & 8 & 33 & 28 & 13 & 16 & 70.22 \\
\hline 13 & 10 & 7 & 37 & 30 & 14 & 57.17 \\
\hline 14 & 37 & 9 & 30 & 7 & 13 & 62.30 \\
\hline 15 & 37 & 7 & 30 & 10 & 13 & 67.28 \\
\hline 16 & 21 & 34 & 31 & 37 & 7 & 83.17 \\
\hline 17 & 14 & 11 & 7 & 28 & 17 & 86.92 \\
\hline 18 & 10 & 7 & 37 & 14 & 31 & 79.19 \\
\hline 19 & 7 & 11 & 34 & 32 & 28 & 98.01 \\
\hline 20 & 10 & 7 & 14 & 27 & 32 & 126.14 \\
\hline 21 & 10 & 37 & 31 & 14 & 7 & 123.80 \\
\hline 22 & 28 & 14 & 7 & 17 & 10 & 118.42 \\
\hline 23 & 9 & 14 & 28 & 32 & 33 & 108.45 \\
\hline 24 & 7 & 37 & 13 & 32 & 9 & 99.78 \\
\hline \multicolumn{6}{|c|}{ Total daily power losses for Scenario 2} & 1996.58 \\
\hline
\end{tabular}

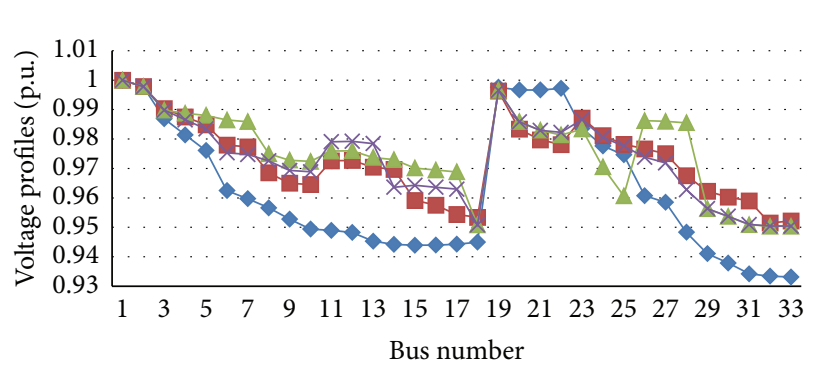

$\rightarrow-$ Voltage for Scenario 1 at $1800 \rightarrow-$ Voltage for Scenario 2 at 1800

$\triangle$ Voltage for Scenario 3 at $1800 \multimap$ Voltage for Scenario 4 at 1800

FIgURE 7: Voltage profiles for all buses for all scenarios with load and PV generation at 1800 .

simple and effective to minimize total daily power loss with no switching required with the optimal daily configuration obtained. Hence, same method can be applied for a year. It also improves the voltage profiles. A 33-bus distribution system with three photovoltaic DGs, different sets of PV generation, and load profiles are successfully used to
TABLE 5: Results of nonsimilar effective configurations matrix obtained in Part 2.

\begin{tabular}{lccccc}
\hline & $S_{1}$ & $S_{2}$ & $S_{3}$ & $S_{4}$ & $S_{5}$ \\
\hline Set switch 1 & 11 & 7 & 27 & 32 & 14 \\
Set switch 2 & 11 & 7 & 28 & 14 & 36 \\
Set switch 3 & 28 & 9 & 33 & 32 & 14 \\
Set switch 4 & 32 & 28 & 34 & 7 & 11 \\
Set switch 5 & 33 & 36 & 10 & 28 & 34 \\
Set switch 6 & 37 & 34 & 9 & 32 & 7 \\
Set switch 7 & 37 & 17 & 9 & 14 & 7 \\
Set switch 8 & 21 & 31 & 37 & 7 & 13 \\
Set switch 9 & 11 & 14 & 30 & 7 & 28 \\
Set switch 10 & 8 & 33 & 28 & 13 & 16 \\
Set switch 11 & 10 & 7 & 37 & 30 & 14 \\
Set switch 12 & 37 & 9 & 30 & 7 & 13 \\
Set switch 13 & 37 & 7 & 30 & 10 & 13 \\
Set switch 14 & 21 & 34 & 31 & 37 & 7 \\
Set switch 15 & 14 & 11 & 7 & 28 & 17 \\
Set switch 16 & 10 & 7 & 37 & 14 & 31 \\
Set switch 17 & 10 & 7 & 14 & 27 & 32 \\
Set switch 18 & 10 & 37 & 31 & 14 & 7 \\
Set switch 19 & 28 & 14 & 7 & 17 & 10 \\
Set switch 20 & 7 & 37 & 13 & 32 & 9 \\
\hline
\end{tabular}

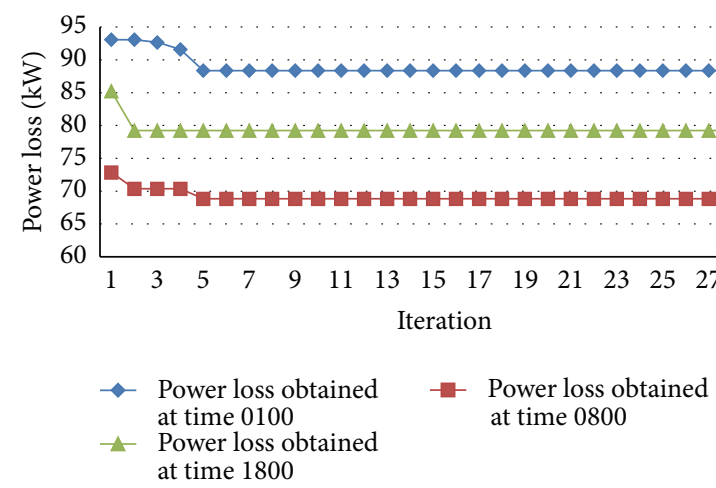

FIGURE 8: Convergence performance of GSA with proposed method at selected time of a day.

demonstrate the effectiveness of the proposed method. A comparison between GSA and EP with selection approach is carried out and the result proven GSA outperforms EP in terms of producing lower total daily power loss.

\section{Conflict of Interests}

The authors declare that there is no conflict of interests regarding the publication of this paper.

\section{Acknowledgments}

The authors thank the University of Malaya and Malaysian Ministry of Education (MOE) for supporting this work through High Impact Research Grant (HIR-MOHE 
D000004-16001) and Universiti Teknologi Malaysia (Q.J130000.2723.00K88).

\section{References}

[1] S. Kalambe and G. Agnihotri, "Loss minimization techniques used in distribution network: bibliographical survey," Renewable and Sustainable Energy Reviews, vol. 29, pp. 184-200, 2014.

[2] S. Civanlar, J. J. Grainger, H. Yin, and S. S. H. Lee, "Distribution feeder reconfiguration for loss reduction," IEEE Transactions on Power Delivery, vol. 3, no. 3, pp. 1217-1223, 1988.

[3] A. González, F. M. Echavarren, L. Rouco, T. Gómez, and J. Cabetas, "Reconfiguration of large-scale distribution networks for planning studies," International Journal of Electrical Power \& Energy Systems, vol. 37, no. 1, pp. 86-94, 2012.

[4] A. Merlin and H. Back, "Search for a minimal-loss operating spanning tree configuration in an urban power distribution system," in Proceedings of the 5th Power System Conference (PSCC '75), Cambridge, UK, 1975.

[5] D. Shirmohammadi and H. W. Hong, "Reconfiguration of electric distribution networks for resistive life losses reduction," IEEE Transactions on Power Delivery, vol. 4, no. 2, pp. 14921498, 1992.

[6] M. E. Baran and F. F. Wu, "Network reconfiguration in distribution systems for loss reduction and load balancing," IEEE Transactions on Power Delivery, vol. 4, no. 2, pp. 1401-1407, 1989.

[7] W.-M. Lin and H.-C. Chin, "A new approach for distribution feeder reconfiguration for loss reduction and service restoration," IEEE Transactions on Power Delivery, vol. 13, no. 3, pp. 870-875, 1998.

[8] J. Zhu, X. Xiong, J. Zhang, G. Shen, Q. Xu, and Y. Xue, "A rule based comprehensive approach for reconfiguration of electrical distribution network," Electric Power Systems Research, vol. 79, no. 2, pp. 311-315, 2009.

[9] A. A. Mohd Zin, A. K. Ferdavani, A. B. Khairuddin, and M. M. Naeini, "Reconfiguration of radial electrical distribution network through minimum-current circular-updatingmechanism method," IEEE Transactions on Power Systems, vol. 27, no. 2, pp. 968-974, 2012.

[10] N. Kokash, An Introduction to Heuristic Algorithms, Department of Informatics and Telecommunications, 2005.

[11] J. Z. Zhu, "Optimal reconfiguration of electrical distribution network using the refined genetic algorithm," Electric Power Systems Research, vol. 62, no. 1, pp. 37-42, 2002.

[12] D. Das, "A fuzzy multiobjective approach for network reconfiguration of distribution systems," IEEE Transactions on Power Delivery, vol. 21, no. 1, pp. 202-209, 2006.

[13] Y. Song, G. Wan, A. Johns, and P. Wang, "Distribution network reconfiguration for loss reduction using fuzzy controlled evolutionary programming," IEE Proceedings-Generation, Transmission and Distribution, vol. 144, no. 4, pp. 345-350, 1997.

[14] J. S. Savier and D. Das, "Impact of network reconfiguration on loss allocation of radial distribution systems," IEEE Transactions on Power Delivery, vol. 22, no. 4, pp. 2473-2480, 2007.

[15] H. Kim, Y. Ko, and K.-H. Jung, "Artificial neural-network based feeder reconfiguration for loss reduction in distribution systems," IEEE Transactions on Power Delivery, vol. 8, no. 3, pp. 1356-1366, 1993.

[16] M. A. Kashem, G. B. Jasmon, A. Mohamed, and M. Moghavvemi, "Artificial neural network approach to network reconfiguration for loss minimization in distribution networks,"
International Journal of Electrical Power and Energy Systems, vol. 20, no. 4, pp. 247-258, 1998.

[17] K. Prasad, R. Ranjan, N. C. Sahoo, and A. Chaturvedi, "Optimal reconfiguration of radial distribution systems using a fuzzy mutated genetic algorithm," IEEE Transactions on Power Delivery, vol. 20, no. 2 I, pp. 1211-1213, 2005.

[18] M. Assadian, M. M. Farsangi, and H. Nezamabadi-Pour, "GCPSO in cooperation with graph theory to distribution network reconfiguration for energy saving," Energy Conversion and Management, vol. 51, no. 3, pp. 418-427, 2010.

[19] B. Enacheanu, B. Raison, R. Caire, O. Devaux, W. Bienia, and N. HadjSaid, "Radial network reconfiguration using genetic algorithm based on the matroid theory," IEEE Transactions on Power Systems, vol. 23, no. 1, pp. 186-195, 2008.

[20] T. Niknam, E. Azadfarsani, and M. Jabbari, "A new hybrid evolutionary algorithm based on new fuzzy adaptive PSO and NM algorithms for distribution feeder reconfiguration," Energy Conversion and Management, vol. 54, no. 1, pp. 7-16, 2012.

[21] C.-T. Su, C.-F. Chang, and J.-P. Chiou, "Distribution network reconfiguration for loss reduction by ant colony search algorithm," Electric Power Systems Research, vol. 75, no. 2-3, pp. 190199, 2005.

[22] Y.-K. Wu, C.-Y. Lee, L.-C. Liu, and S.-H. Tsai, "Study of reconfiguration for the distribution system with distributed generators," IEEE Transactions on Power Delivery, vol. 25, no. 3, pp. 1678-1685, 2010.

[23] R. Srinivasa Rao, S. V. L. Narasimham, M. Ramalinga Raju, and A. Srinivasa Rao, "Optimal network reconfiguration of largescale distribution system using harmony search algorithm," IEEE Transactions on Power Systems, vol. 26, no. 3, pp. 10801088, 2011.

[24] K. S. Kumar and T. Jayabarathi, "Power system reconfiguration and loss minimization for an distribution systems using bacterial foraging optimization algorithm," International Journal of Electrical Power \& Energy Systems, vol. 36, no. 1, pp. 13-17, 2012.

[25] J. S. Savier and D. Das, "Impact of network reconfiguration on loss allocation of radial distribution systems," IEEE Transactions on Power Delivery, vol. 22, no. 4, pp. 2473-2480, 2007.

[26] M. M. Aman, G. B. Jasmon, H. Mokhlis, and A. H. A. Bakar, "Optimal placement and sizing of a DG based on a new power stability index and line losses," International Journal of Electrical Power \& Energy Systems, vol. 43, no. 1, pp. 1296-1304, 2012.

[27] Z. Yasin and T. Rahman, Network Reconfiguration in a Power Distribution System under Fault Condition with the Presence of Distributed Generation, Universiti Tenaga Nasional, Selangor, Malaysia, 2006.

[28] J. Olamaei, T. Niknam, and G. Gharehpetian, "Application of particle swarm optimization for distribution feeder reconfiguration considering distributed generators," Applied Mathematics and Computation, vol. 201, no. 1-2, pp. 575-586, 2008.

[29] W. M. Dahalan, H. Mokhlis, A. H. A. Bakar, and J. J. Jamian, "The simultaneous application of optimum network reconfiguration and distributed generation sizing using PSO for power loss reduction," Przeglad Elektrotechniczny, vol. 89, no. 4, pp. 137-141, 2013.

[30] M. M. Aman, G. B. Jasmon, A. H. A. Bakar, and H. Mokhlis, "A new approach for optimum simultaneous multi-DG distributed generation Units placement and sizing based on maximization of system loadability using HPSO (hybrid particle swarm optimization) algorithm," Energy, vol. 66, pp. 202-215, 2014.

[31] Y. HuPing, P. YunYan, and X. Ning, "Gradual approaching method for distribution network dynamic reconfiguration," in 
Proceedings of the Workshop on Power Electronics and Intelligent Transportation System (PEITS '08), pp. 257-260, IEEE, Guangzhou, China, August 2008.

[32] A. E. Milani and M. R. Haghifam, "An evolutionary approach for optimal time interval determination in distribution network reconfiguration under variable load," Mathematical and Computer Modelling, vol. 57, no. 1-2, pp. 68-77, 2013.

[33] T. Hao, L. Lin, and W. Yanhan, "Research of distribution network reconfiguration with renewable energy power generation unit," in Proceedings of the IEEE International Conference on Power System Technology (POWERCON '14), pp. 2680-2685, IEEE, Chengdu, China, October 2014.

[34] A. S. Bouhouras, T. A. Papadopoulos, G. C. Christoforidis, G. K. Papagiannis, and D. P. Labridis, "Loss reduction via network reconfigurations in Distribution Networks with Photovoltaic Units Installed," in Proceedings of the 10th International Conference on the European Energy Market (EEM '13), pp. 1-8, Stockholm, Sweden, May 2013.

[35] E. Rashedi, H. Nezamabadi-pour, and S. Saryazdi, "GSA: a gravitational search algorithm," Information Sciences, vol. 179, no. 13, pp. 2232-2248, 2009.

[36] H. Yang, F. Wen, and L. Wang, "Newton-Raphson on power flow algorithm and Broyden method in the distribution system," in Proceedings of the IEEE 2nd International Power and Energy Conference (PECon '08), pp. 1613-1618, December 2008.

[37] B. Shaw, V. Mukherjee, and S. P. Ghoshal, "A novel oppositionbased gravitational search algorithm for combined economic and emission dispatch problems of power systems," International Journal of Electrical Power and Energy Systems, vol. 35, no. 1, pp. 21-33, 2012.

[38] Y. M. Atwa, E. F. El-Saadany, M. M. A. Salama, and R. Seethapathy, "Optimal renewable resources mix for distribution system energy loss minimization," IEEE Transactions on Power Systems, vol. 25, no. 1, pp. 360-370, 2010.

[39] N. H. B. Jalal, Operating Code 1: Demand Forecast, The Malaysian Grid Code Awareness Programme, Kuala Lumpur, Malaysia, 2014. 


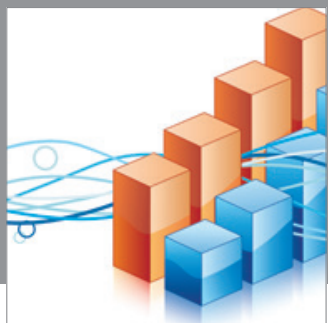

Advances in

Operations Research

mansans

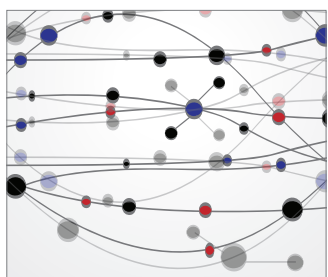

The Scientific World Journal
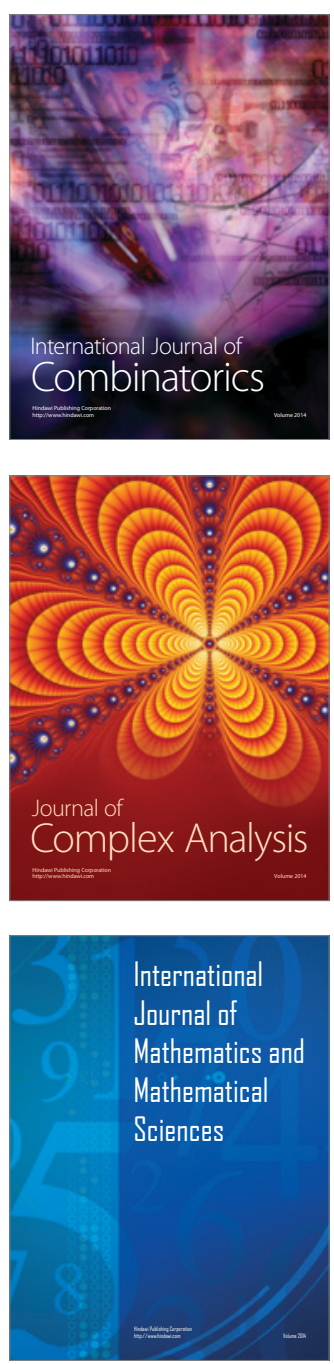
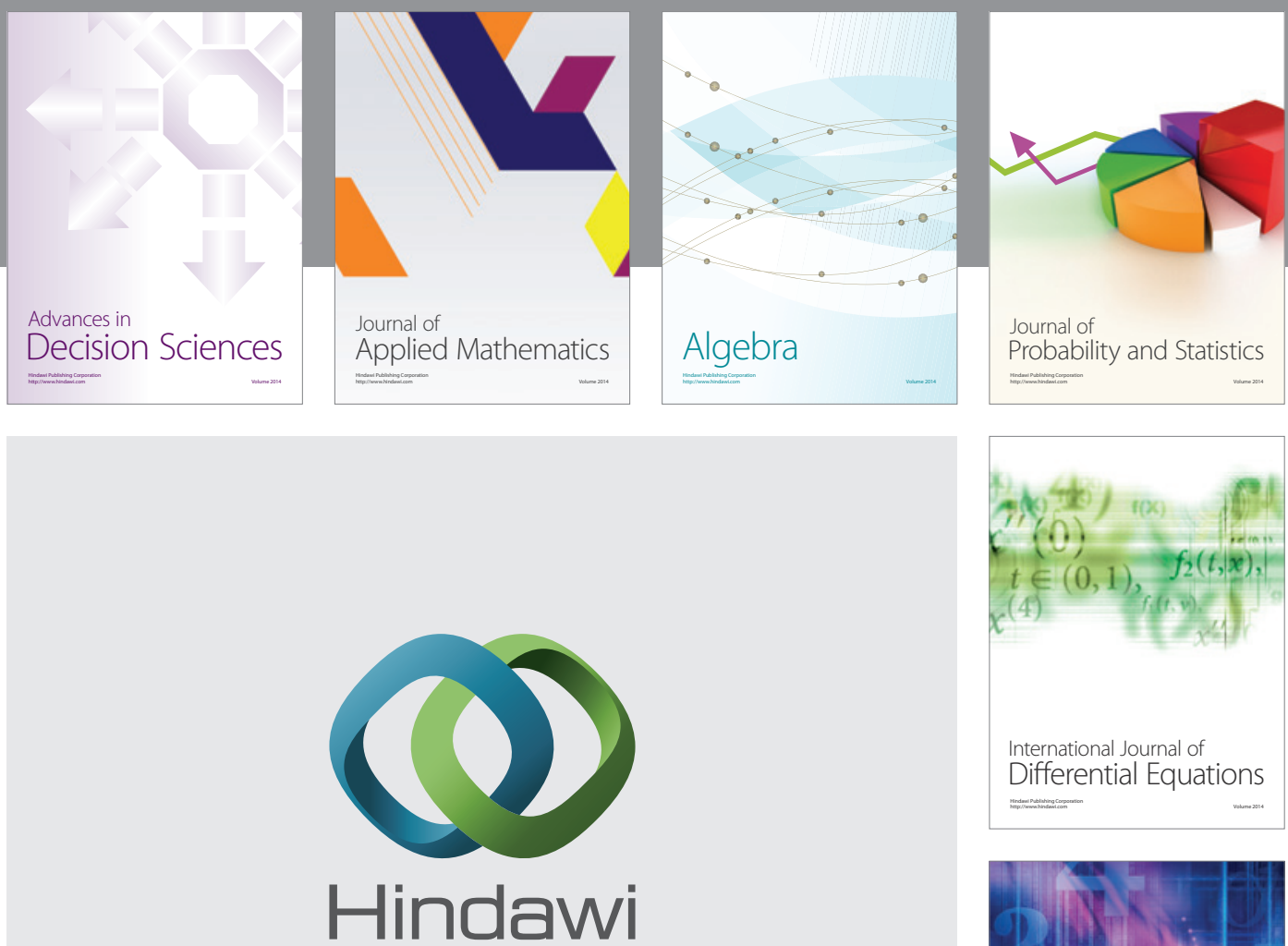

Submit your manuscripts at http://www.hindawi.com
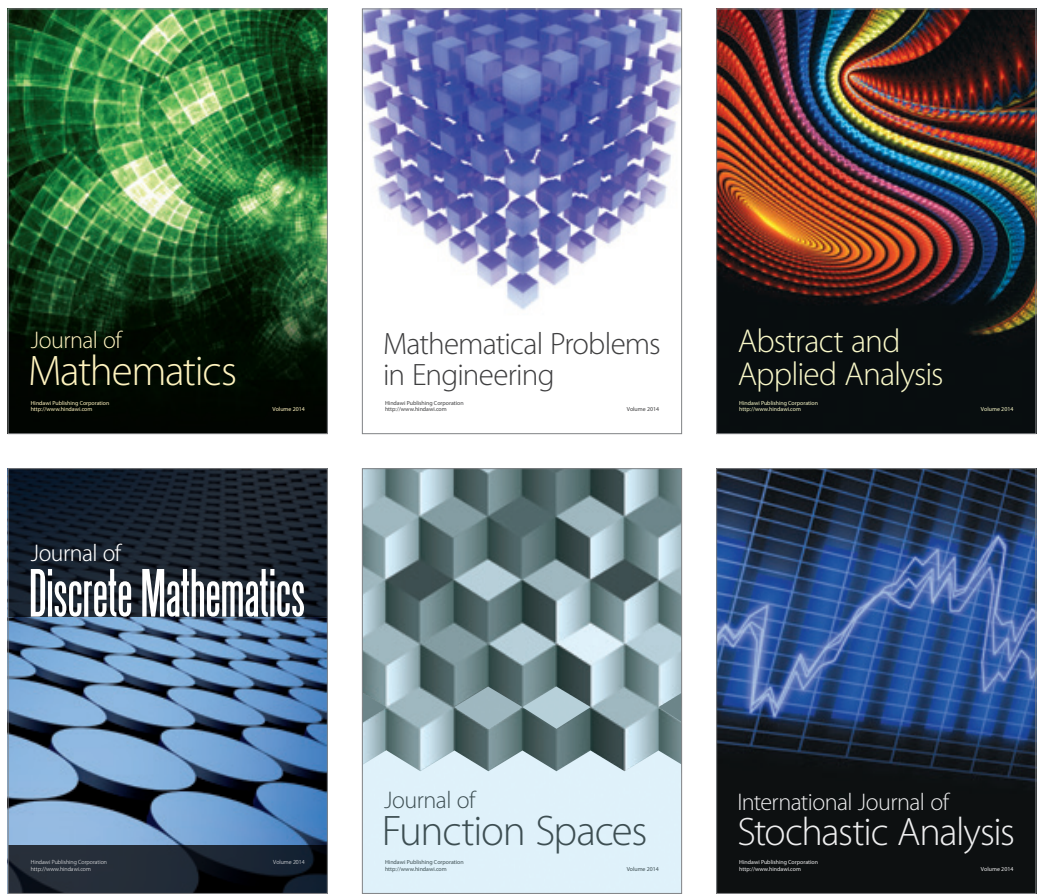

Journal of

Function Spaces

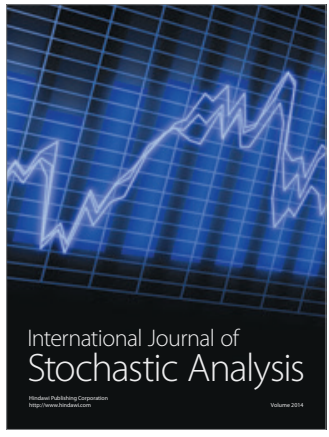

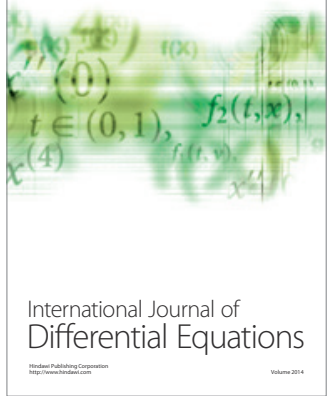
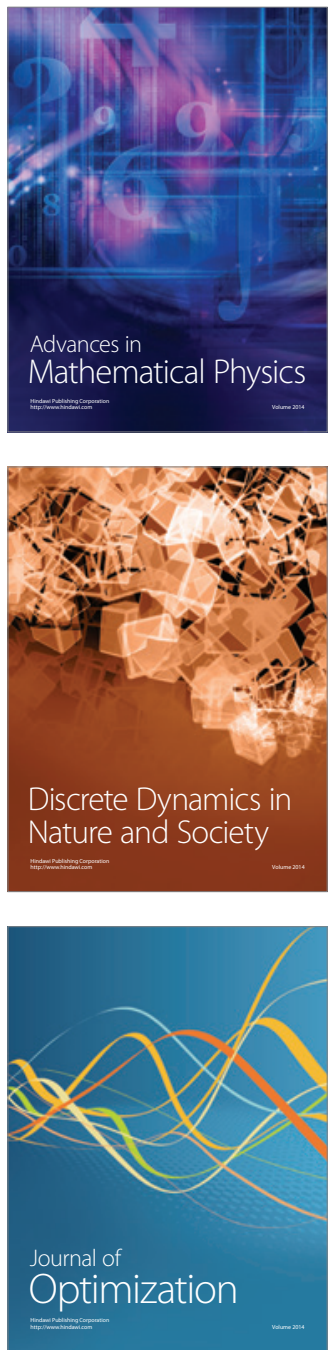